\title{
Integrating Lifestyle Focused Approaches into the Management of Primary Dysmenorrhea: Impact on Quality of Life
}

This article was published in the following Dove Press journal: International Journal of Women's Health

\author{
Orestis Tsonis $\mathbb{D}^{1}$ \\ Fani Gkrozou ${ }^{2}$ \\ Zoi Barmpalia (1D) \\ Annamaria Makopoulou ${ }^{3}$ \\ Vassiliki Siafaka ${ }^{4}$ \\ 'Department of Obstetrics and \\ Gynaecology, University Hospital of \\ loannina, loannina, Greece; ${ }^{2}$ Department \\ of Obstetrics and Gynaecology, \\ University Hospitals of Birmingham, \\ Birmingham, UK; ${ }^{3}$ School of Psychology, \\ College of Human Sciences, Bangor \\ University, Bangor, Wales, UK; \\ ${ }^{4}$ Department of Speech and Language \\ Therapy, School of Health Sciences, \\ University of Ioannina, loannina, Greece
}

Objective: This narrative review aims to identify alternative ways to improve the symptoms of primary dysmenorrhea (PD).

Background: PD refers to endometrial painful cramps during the premenstrual period. This condition affects a lot of women worldwide and is accompanied with absenteeism and high economic costs, thus, risk-free, and effective therapeutic approaches are needed. Pharmacological agents such as non-steroidal anti-inflammatory drugs (NSAIDs) and oral contraceptive pills (OCPs), which are widely prescribed for these women, demonstrate adequate efficacy in alleviating pain and discomfort. The long natural course of the disease dictates remedies that focus on lifestyle changes and on improvement of Quality-of-Life (QoL) for women suffering with PD.

Materials and Methods: Five major search engines, namely MEDLINE, PubMed, EMBASE, and Cochrane Library were searched for articles published prior to October 2020 focused in PD. A total of 74 paper were included.

Discussion: Physical activity, for instance yoga, aromatherapy massage, and other forms of relaxation, vitamins and dietary changes, acupressure and acupuncture, a and some psychological interventions are just few of the proposed health behavior targeted approaches in cases of PD. This review focuses on lifestyle changes and alternative methods that could potentially result in minimizing symptoms of PD and in improving overall QoL for these patients, by providing current scientific evidence on their efficacy.

Conclusion: Complementary and alternative medicine practices (CAM) are widely accepted by women. International literature provides controversial scientific evidence, thus further studies need to be conducted in order to prove or disregard their efficacy in cases of PD.

Keywords: primary dysmenorrhea, quality-of-life, therapy, complementary and alternative medicine, pain relief

\section{Introduction}

Primary dysmenorrhea (PD) is recurrent menstrual cramps during the menstrual period and painful menses that are not associated with any other obvious medical pathology, with an indigenous sensation of pain, a few days prior to menstruation, that normally persists approximately, 48-72 hours after the onset of menstrual bleeding. ${ }^{1}$ PD is considered the commonest complaint for adolescents as well as, women of reproductive age, and this condition manifests through a wide variety of symptoms, either physical or affective, ${ }^{2}$ such as back pain, abdominal and pelvic pain, headaches, migraines, insomnia, nausea, dizziness, fatigue, sweating, cramps, irritability,
Correspondence: Orestis Tsonis Senior Registrar in Obstetrics and Gynaecology, Department of Obstetrics and Gynaecology, University Hospital of loannina, Stavrou Niarchou Avenue, loannina, 45500, Greece Tel +306986620604

Email orestis.tsonis@gmail.com

International Journal of Women's Health 2021:13 327-336 
depression, and emotional instability, with a negative impact on overall Quality-of-Life (QoL) compared to healthy women. ${ }^{1,3}$ International literature lacks consistent scientific evidence with regards to PD's prevalence, reporting various trends of between $45-95 \%$ due to different methods of measurements and definitions used worldwide. ${ }^{1,4}$

This condition is postulated to arise from an abundant production of indigenous prostaglandins that result in an irregular pattern of endometrial contractions along with a decrease in the overall endometrial blood supply. Current medical agents against PD have been well established, such as NSAIDs (Non-Steroidal Anti-Inflammatory Drugs) and COPs (Combined Oral Contraceptives), and act in a way that minimizes the production of prostaglandins. Unfortunately, these drugs come with unpleasant side-effects, such as nausea, vomiting, GI bleeding, acne, and asthma, to name a few, especially when administered for long-term use. ${ }^{3}$

The long physical course of PD demands therapeutic approaches that regard complementary medications and a holistic management for these patients without jeopardizing patient's overall QoL. Patients ought to adopt novel lifestyle targeted habits with regards to nutrition, physical activity, and complementary practices that minimize the effects of PD while improving QoL. ${ }^{3}$ That said, absenteeism at school and work, which is clearly linked with increased financial cost for society, make this lifestyle targeted change imperative for these patients in order to establish an approach that could relieve individuals suffering from PD by maintaining highest therapeutic efficacy along with improved QoL.

Over the last decades researchers have studied health behaviors change in a wide variety of conditions. Achieving a change in a person's health behaviors is a difficult and complex process. Consequently, the deviations vary and may have a significant impact on the individual's health and on the health system. Theories for understanding and enhancing health beliefs (eg, Health Belief Model, Health Promotion Model, and Theory of Reasoned Action) argue that it is relatively easier to achieve onset, than to maintain it in the long-term. The pattern of health behavior change seems to be common, with high recurrence rates varying early after the change. In addition, social factors influence behavior, with an ambiguous impact on both initiating and maintaining health behavior change. ${ }^{5}$

In the literature, a plethora of complementary and alternative medicine (CAM) practices and medications have been suggested, but the efficacy of these suggestions remain still controversial. ${ }^{6}$ Similarly as in the case of postmenopausal symptoms, neutraceutical alternates have been suggested. Women that refuse hormonal replacement treatment (HRT) can potentially use Isoflavones, herbal derivatives, vitamins, and other compounds such as polyphenols. ${ }^{7}$ Over the last 10 years, CAM therapies have been proposed, especially in regions of Africa and Asia where traditional medicine remains trusted by the majority of patients, therefore, approximately all studies conducted regarding CAM efficacy originated from these countries. $^{2,8,9}$ Physical activity, for instance yoga, aromatherapy, psychotherapy, and other forms of relaxation, vitamins and dietary changes, acupressure and acupuncture are just few of the proposed lifestyle targeted therapies in cases of $\mathrm{PD}^{3}$ This review focuses on lifestyle changes that could potentially result in minimizing symptoms of PD and in improving overall QoL for these patients, by providing current scientific evidence on their efficacy.

\section{Materials and Methods}

Five major search engines, namely MEDLINE, PubMed, EMBASE, and Cochrane Library, were searched for articles published prior to October 2020 that matched any combination of the following key words: [primary dysmenorrhea] OR [dysmenorrhea] OR [menstrual pain] AND [life-style] OR [alternative medicine] OR [complementary medicine] OR [diet] OR [physical activity] OR [herbs] OR [acupressure] OR [acupuncture] OR [aromatherapy] OR [heat therapy] AND [emotional support] AND [psychological support] AND [psychotherapy]. Only scientific papers in English were included. A total of 74 papers were included.

This paper presents the results of a narrative review. The review focuses on lifestyle modifications that alleviate painful symptoms in patients suffering from Primary Dysmenorrhea. All complementary and alternative therapeutic approaches focusing on lifestyle changes, that are supported by valid scientific evidence, are presented.

These are nutrition, physical activity, Body Mass Index (BMI), herbs, essential oils, and medical plants. In addition, less explored approaches, the relevant sociocultural issues, and psychological aspects are mentioned, in order to present all the available information in the literature. Acupressure, acupuncture, and reflexology are also mentioned, since these methods are alternative types of medicine which represent a different lifestyle and encourage 
people to differ their everyday life and habits. They are based on a different philosophy and approach than the general western lifestyle.

Papers addressing secondary dysmenorrhea or other evident pathologies that could cause menstrual pain, such as endometriosis, have been excluded.

\section{Nutrition}

Maintaining a healthy diet containing dairy products and fibre has been postulated to positively act against PD symptoms. ${ }^{1}$ Salt and animal fat restrictions have also been proposed as effective for this condition. ${ }^{10}$ Interestingly, skipping breakfast is linked with $\mathrm{PD}$, while overall low calorie intake has been found to be a risk factor for this condition. ${ }^{1}$ A possible pathophysiologic explanation is that a normal $\mathrm{BMI}$ is associated with regular ovulation and menses, whereas extreme BMIs lead to a prostaglandin abundance that causes painful menstruation. Scientific evidence remains inconclusive, and further studies need to be conducted in order to check the validity of such hypotheses.

Warm beverages and food were found to be preferred by women suffering from PD in order to alleviate their symptoms. Moreover, women with PD with a family history of PD in their mothers were more likely to adopt a healthier nutrition plan, suggesting that PD awareness might increase lifestyle focused approaches into the management of Primary Dysmenorrhea. ${ }^{11}$ Reduction in beverages containing caffeine was also a nutritional modality that was adopted by patients with the disease, as well as meat restriction, in order to minimize the unpleasant impact of PD in their QoL. ${ }^{4}$

A 2016 Cochrane review study revealed the insufficiency of scientific evidence so far, and, while vitamin E supplements are not considered effective, fish oil and vitamin B1 evidence is very limited to safely draw a conclusion about their use in cases of $\mathrm{PD} .^{12}$

\section{Physical Activity and BMI}

With regards to body fitness, maintaining a stable Body Mass Index (BMI), between $19-24.9 \mathrm{~kg} / \mathrm{m}^{2}$, is associated with lower rates of $\mathrm{PD}$, whereas weight extremes exacerbate painful symptoms in patients with $\mathrm{PD}$, as a recent Chinese cross-sectional study suggests. ${ }^{1}$

Yoga is considered an excellent tool in reducing pain duration and severity in cases of PD. ${ }^{13}$ More specifically, women with PD are instructed to follow a 20-minute session daily during their luteal phase, emphasizing in cobra, cat, and fish postures to alleviate their painful symptoms. ${ }^{14}$ Yoga intervention was associated with improved QoL and decreased physical pain and discomfort. ${ }^{15,16}$ More specifically, patients with PD who trained by yoga for at least 6 months were more likely to reporthigher levels of energy and concentration, as well as lower occurrence of sleep disturbances, insomnia, and confusion. These patients demonstrated stress relief, more social relationships, and higher work capacity by participating actively in their daily activities. ${ }^{15}$

Other forms of physical activity, such as isometric exercise and stretching, running, Kegel exercises, and relaxation postures have also been proposed to release pain and discomfort in dysmenorrhea, when performed regularly. ${ }^{17-19}$ It seems that 50 minutes of exercise at least 3-times per week has a positive impact in women suffering from painful menses. Physical activity is an excellent tool in maintaining a healthy body, normal cardiovascular function, and improved emotional balance, while physical inactivity is considered the fourth most important risk factor of mortality worldwide as it is strongly linked with cancer, diabetes, and cardiovascular disease. ${ }^{18}$ Aerobic activity results in an indigenous release of opioids that improve overall QoL and reduce pain sensitivity in women with PD. ${ }^{18}$

\section{Herbs, Essential Oils, and Medical Plants}

A wide variety of herbs has been suggested to improve symptoms in patients suffering from PD. Chamomile, fennel, cinnamon, and aloe vera are only a few of the herbal regimens that are proposed to treat the severity of symptoms by their anti-inflammatory and anti-spasmodic properties. $^{3}$

Chamomile is widely used in European traditional medicine for its analgesic and anti-inflammatory effects. A number of studies have suggested its action on the uterus by increasing blood flow and minimizing pain symptoms for patients with PD. Chamomile has been proven to treat cases of migraines and muscle pain, thus, its potential therapeutic effect on dysmenorrhea is quite possible. Matricaria chamomilla minimizes mood swings, anger, and depression, as well as pain perceived severity, fatigue, and lethargy compared to control group patients that received no medication. ${ }^{12,20}$

Fennel originated by Umbelliferae contains anethole, fenchone, and limonene, which act as dopamine-like agents. ${ }^{21}$ Anethole blocks dopamine receptors and inhibits endometrial contractions caused mainly by 
prostaglandins. ${ }^{3}$ Thus, fennel supplements decrease pain perception in these patients. Along with the analgesic properties of this plant, mainly used as a herb in Chinese culture, the lack of side-effects during its use make it ideal therapy in cases of PD, where long-term treatment is required. Foeniculum vulgar Mill, publicly known as fennel, lowers pain scores in patients with PD and improves overall QoL. ${ }^{12,22}$

Zataria multiflora is another commonly used herb in patients suffering from PD due to its inhibitory effect against prostaglandin production. More specifically, thymol, as the main ingredient of this plant, blocks calcium channels that are responsible for endometrial cramps. ${ }^{12}$ The effect of Zataria multiflora oil has been suggested to minimize the severity of pain in women with PD as well as to shorten the duration of pain. ${ }^{12,23,24}$

Other herbs, such as ginger (Zingiber officinale), ${ }^{25}$ Valeriana officinalis, ${ }^{21}$ Cinnamomum (cinnamon), ${ }^{26}$ and lavender oil, ${ }^{27}$ provide pain intensity reduction equal to pharmacological agents, such as mefenamic acid, although their exact therapeutic mechanism remains unclear. ${ }^{20,28-36}$ A 2016 Cochrane 2016 emphasized the existence of limited evidence so far in herbal medicine and proposed further RCTs in order to assess the overall effect of herbal medicine in cases of PD. ${ }^{12}$ Research in the international literature shows dozens of herbal plants used in PD with promising results. Thus, well-designed RCTs should be employed in order to provide valid scientific evidence on their efficacy since, so far, the findings are inconsistent.

\section{Acupressure, Acupuncture, and Reflexology}

In cases of $\mathrm{PD}$, neuromuscular manipulation and acupressure have been adopted by many patients who consistently report a meaningful improvement in pain intensity and duration. ${ }^{37}$ Although of low quality, the majority of studies report the effectiveness of this CAM modality in patients suffering from $\mathrm{PD} .^{38}$ Many sites have been proposed, while auricular acupressure gained much popularity due to the simplicity of self-therapy. ${ }^{38}$ Acupressure by trained personneldemonstrates higher rates of efficacy among studies, ${ }^{39,40}$ but self-administered acupressure remains a risk-free and efficient CAM modality in patients with dysmenorrhea. ${ }^{39,41}$ The effect of acupressure has also been proven in cases of obesity, smoking cessation, acne, and emotional stability, but the exact mechanism of action remains unclear. ${ }^{42}$ In cases of PD though, patients who received acupressure were found to have increased uterine blood flow that potentially minimized the pain and discomfort experienced during menses. ${ }^{38}$ Many patients adopt manipulative therapy as an adjunct therapeutic tool in the management of PD. ${ }^{43} \mathrm{~A}$ recent RCT that investigates the effect of acupressure in PD concluded that manipulating menstrual pain zones during $10-15$ sessions provided pain relief for patients with PD along with high rates of satisfaction. ${ }^{44}$ The exact therapeutic duration of each manipulation though needs to be further explored, as international literature fails to present long-term reduction of painful symptoms for these patients. ${ }^{45,46}$

Many studies so far have praised the effect of acupuncture in minimizing the pain of PD..$^{39,47,48}$ The evidence shows that acupuncture reached higher overall improvement of pain in these patients compared to NSAIDs monotherapy. ${ }^{19,47,49}$ Unfortunately, despite the success noted by the vast majority of studies on the subject, the Cochrane Database of Systematic Review found very low quality of evidence, mainly attributed to risk of bias, poor reporting, inconsistency, and risk of publication bias. ${ }^{47} \mathrm{In}$ terms of QoL in women with PD that receive acupuncture alternative therapy, the evidence shows improved physical function, mental health, bodily pain, vitality, social function, and emotional role, while no adverse outcomes by this intervention were reported. ${ }^{3,50}$ Nevertheless, authors suggest 20-minutes of acupuncture per day applied for half the days of a month during a trimester as effective in reducing dysmenorrhea. ${ }^{48,51}$ The ost common needle sites for effective acupuncture in these cases, that have been proposed by the international literature, are SP6, BL54, and ST28. ${ }^{52,53}$

Aromatherapy massage has also been suggested as beneficial in minimizing pain and discomfort in patients with PD. During aromatherapy massage, essential oils are applied directly to the skin, or during a bath, or they are inhaled. Abdominal massage follows as the essential oils, mainly lavender, rose damascene, cinnamon, and clove, penetrate deep within the tissues. A recent metanalysis ${ }^{54}$ found that aromatherapy massage with the use of these essential oils is superior to placebo oils in reducing pain perception and discomfort in women with PD. ${ }^{55}$ More specifically, lavender oil was found to be better in alleviating painful symptoms in these patients compared to other mixed essential oils. ${ }^{27,54}$ Another meta-analysis by Song et al ${ }^{56}$ confirmed the positive effect of aromatherapy in reducing dysmenorrhea, but the authors underlined the high diversity and heterogeneity 
among studies that could potentially increase the risk of bias.

\section{Less-Explored Approaches on the Management of PD}

Despite the traditional approaches in use, a wide range of futuristic, novel approaches on the management of PD have been documented. Some show evidence of a possible positive effect on painful menses, while others, although widely used, are largely abandoned for this condition, such as homeopathy, due to limited scientific data. $^{57}$

Hydrotherapy and heat therapy have been proposed as beneficial in pain reduction in cases of PD. ${ }^{58}$ With regards to hydrotherapy, warm baths and hip/sitz baths have been speculated as therapeutic for painful menses, while Heat patches containing iron chips have also been suggested to provide relief of PD discomfort equal to ibuprofen. ${ }^{59}$ Nevertheless, a recent systematic review and metanalysis revealed only little-to-moderate pain reduction in heat therapy. ${ }^{19}$ In other words, the role of naturopathic medicine in general might be subjective and it remains solely down to the woman's initiative whether she uses such modalities. Although these practices have existed in European traditional culture since the 18th century, their contemporary use is limited.

Kinesio taping along with body awareness have demonstrated favorable outcomes with regards to minimizing pain during menses in women with PD, but the evidence is not enough to support its use widely. Similar, in that cases more studies are necessary to support or not its efficacy. ${ }^{10}$ In patients with chronic pain such as PD, studies have shown a distorted body awareness and body sensitivity, thus physiotherapy of any form (kinesio taping, electrotherapy, spinal manipulation, heat therapy, etc.) increases body awareness. The effect size of these interventions, though, is yet to be estimated in future studies. ${ }^{10}$

Another peculiar approach, in treating symptoms of PD is low-light laser level skin-adhesive therapy (LLLT), reaching a reduction of $83 \%$ compared to a placebo, as a randomized, double-blind, placebo-controlled pilot trial suggests. ${ }^{60}$ Light properties in cases of PD have also been tested with near-infrared belts used by these patients. Authors have suggested that the warmth produced by these tools improves abdominal blood supply and their efficacy can be safely compared to the use of oral analgesics. $^{61}$

\section{Sociocultural Issues and Health Behaviors Related to PD}

A number of lifestyle modifications has been found to alleviate painful symptoms accompanying $\mathrm{PD}$, which should be examined under a cultural context. ${ }^{11}$ In other words, Asians and Africans were more likely to trust traditional medicine and to opt into a lifestyle that includes herbs and medicinal plants. In a recent Chinese crosssectional study, adolescents with PD were more like to reduce physical activity and to adopt heat therapy by using warmer clothes during painful menses. Furthermore, these patients were more likely to change their diet by increasing their warm beverage and food intake. Also, these patients reported low levels of self-meditation, but were more likely to express their negative emotions due to PD to their peers in order to seek emotional support. ${ }^{11}$ On the other hand, western cultures were more likely to seek medical advice and to use pharmacological agents in order to minimize their painful symptoms and discomfort. ${ }^{4}$ Interestingly, a recent Malaysian study demonstrated that the need to reduce pharmacological agents was independent to the use of CAM modalities, but CAM users found that the absence of side-effects in CAM therapies improved their QoL $^{62}$ (Figure 1).

Alcohol consumption and smoking is reported as a risk factor for developing PD. ${ }^{1,4}$ Unfortunately, at this time the scientific evidence does not quantify alcohol consumption, smoking, or environmental tobacco smoke for patient with PD. ${ }^{4}$ In general, although no threshold can be estimated, potentially smoking cessation and overall alcohol withdrawal are wise strategies to improve their QoL regardless of the existence of underlying PD.

Socio-economic status seems to play a crucial role in young adolescents during first years of university, when various studies report that the symptoms of PD are accompanied by an extreme severity. ${ }^{1}$ Possibly, different cultural and religious beliefs seem to exacerbate painful feelings of dysmenorrhea that are exacerbated among peers of different racial backgrounds, as it is usually the case in universities. Women of families of lower monthly income present with severe menstrual pain, a fact that might be explained by the inability to seek medical attention for their condition. ${ }^{1}$

\section{Psychological Aspects}

The correlation between psychological factors and primary dysmenorrhea has been thoroughly described in the 


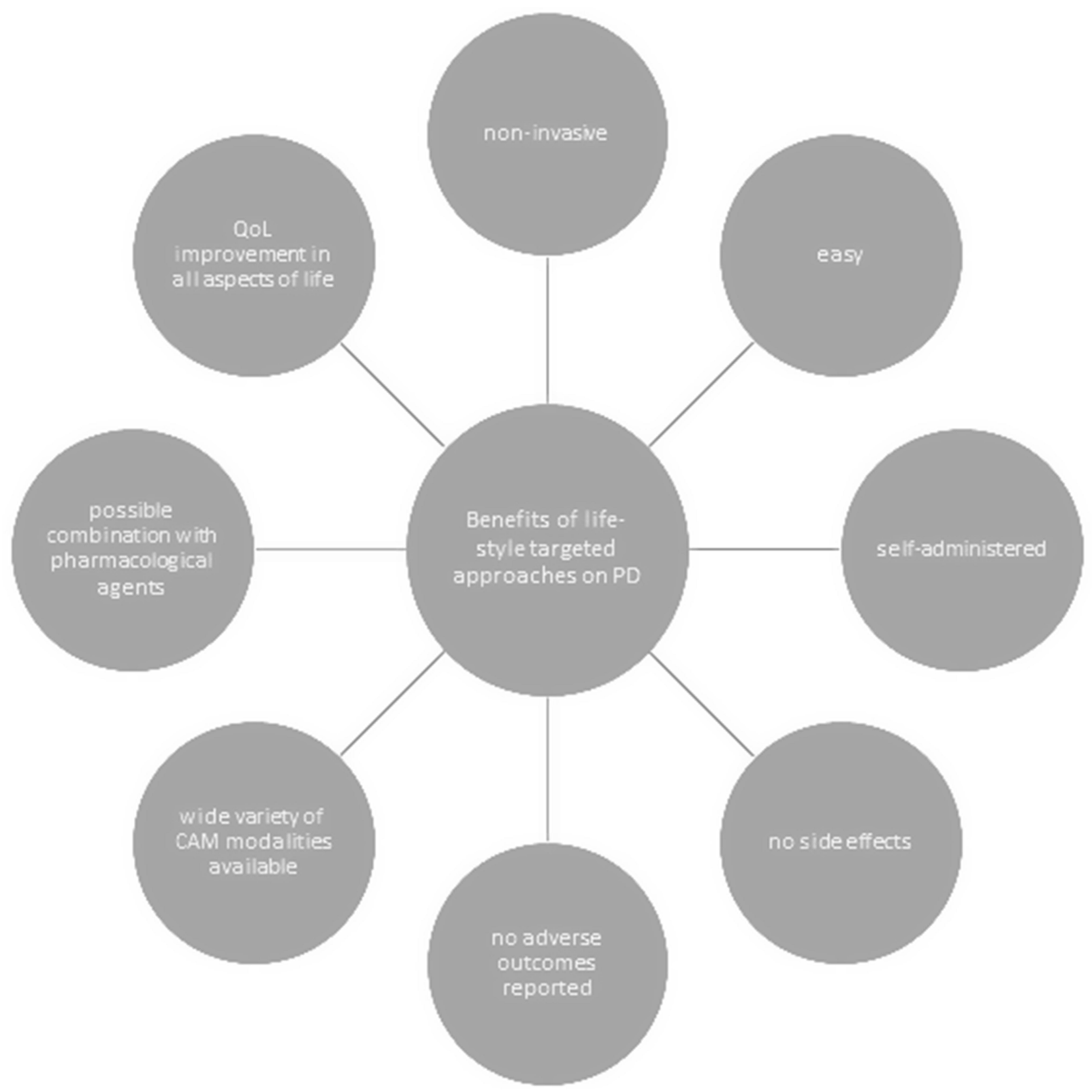

Figure I Benefits of lifestyle targeted approaches on the management of PD.

Abbreviations: PD, primary dysmenorrhea; CAM, complementary and alternative medicine; QoL, quality-of-life.

literature. ${ }^{63,64}$ The experience of recurrent menstrual pain increases the risk of mental disorders and especially stress, anxiety, and depression, but also alcohol abuse and somatic symptoms disorders. ${ }^{64}$ In addition, the comorbidity of dysmenorrhea and depression may contribute to an increased focus on symptoms and by extent on the perceived intensity of pain. ${ }^{63,65}$

The tendency to maximize negative thoughts about pain is known as pain catastrophizing and seems to be associated with the frequency and intensity of pain and emotional distress, as well as with the consequent decreased functionality. Research suggests that women with dysmenorrhea report high levels of catastrophizing pain, ${ }^{66}$ as well as increased pain intensity, lower perceived effectiveness of over-the-counter drugs, and significant lack of functionality. ${ }^{67}$ Although it remains unclear whether pain catastrophizing is a personality trait or a coping strategy, it seems to be associated with the adoption of poor coping strategies both cognitively (eg, distraction and imagery) and behaviorally (eg, reduced activity). ${ }^{67-69}$ 
It is widely accepted that the experience of pain should be considered through an interdisciplinary approach. Support programs, and in particular those including the Cognitive Behavioral Therapy (CBT), appear to be effective in increasing knowledge about primary dysmenorrhea and reconstructing dysfunctional cognition with more rational ones, as well as increasing self-regulation skills. These interventions result in a better understanding of the meaning of pain, decrease of symptoms, and reduction of analgesic use. ${ }^{70,71}$ Well-designed studies are needed for a deeper understanding of the relationship between common mental health conditions and primary dysmenorrhea that will ensure a better clinical approach.

Since it has been well-established that body image awareness and general health perception affect the intensity and duration of pain for these women, trained specialists should promote a more holistic approach by integrating lifestyle changes into the management of primary dysmenorrhea. ${ }^{62,72,73}$ Lifestyle alterations could benefit the overall health of these women and improve their Quality-of-

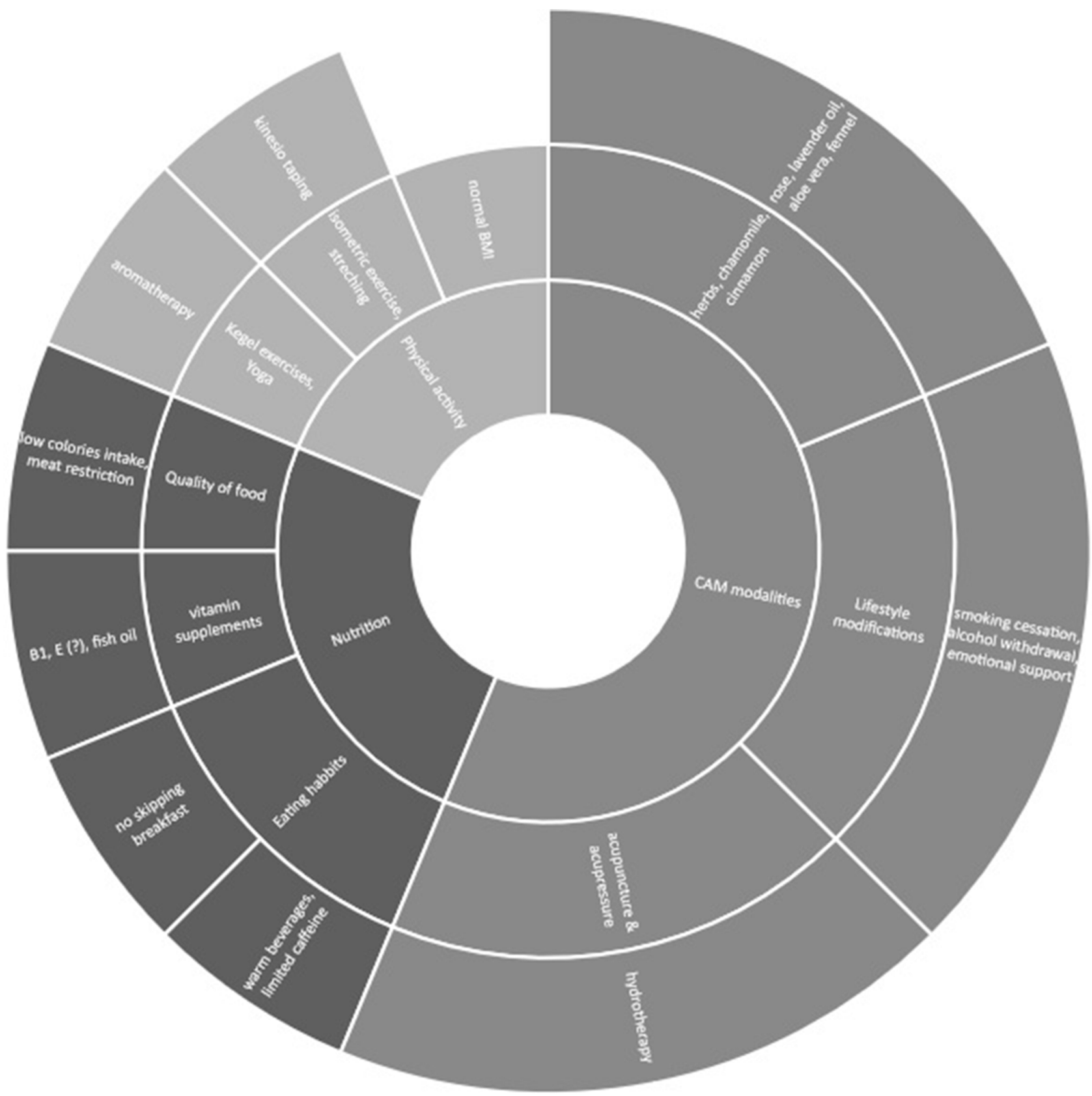

Figure 2 Integrating lifestyle focused approaches into the management of PD.

Abbreviations: BMI, body mass index; CAM, complementary and alternative medicine. 
Life. $^{74}$ Figure 2 comprises the aforementioned lifestyle focused approaches into the management of PD.

\section{Conclusions}

CAM modalities seem to be widely accepted and adopted by women in adolescence or of reproductive age affected with perimenstrual pain and discomfort. International literature provides controversial scientific evidence with regards to their benefits, while the majority of them hide undocumented risks.

CAM approaches are non-pharmacological and promise to offer relief and to reduce side-effects. These CAM remedies are often self-prescribed with little or no professional caregiver's oversight, thus further studies need to be conducted in order to prove or disregard their efficacy in cases of PD.

\section{Disclosure}

All authors declare no conflicts of interest nor funding of any kind.

\section{References}

1. Hu Z, Tang L, Chen L, Kaminga AC, Xu H. Prevalence and risk factors associated with primary dysmenorrhea among Chinese female university students: a cross-sectional study. J Pediatr Adolesc Gynecol. 2020;33(1):15-22. doi:10.1016/j.jpag.2019.09.004

2. Fisher C, Sibbritt D, Hickman L, Adams J. A critical review of complementary and alternative medicine use by women with cyclic perimenstrual pain and discomfort: a focus upon prevalence, patterns and applications of use and users' motivations, information seeking and self-perceived efficacy. Acta Obstet Gynecol Scand. 2016;95 (8):861-871. doi:10.1111/aogs.12921

3. Sharghi M, Mansurkhani SM, Ashtary-Larky D, et al. An update and systematic review on the treatment of primary dysmenorrhea. $J$ Bras Reprod Assist. 2019;23(1):51-57. doi:10.5935/1518-0557.20180083

4. Fernández-Martínez E, Onieva-Zafra MD, Laura Parra-Fernández M. Lifestyle and prevalence of dysmenorrhea among Spanish female university students. PLoS One. 2018;13(8):1-11. doi:10.1371/journal. pone. 0201894

5. Lippke S, Ziegelmann JP. Theory-based health behavior change: developing, testing, and applying theories for evidence-based interventions. Appl Psychol. 2008;57(4):698-716. doi:10.1111/j.14640597.2008.00339.x

6. Fisher C, Adams J, Hickman L, Sibbritt D. The use of complementary and alternative medicine by 7427 Australian women with cyclic perimenstrual pain and discomfort: a cross-sectional study. BMC Complement Altern Med. 2016;16(1):1-11. doi:10.1186/s12906-016-1119-8

7. De Franciscis P, Colacurci N, Riemma G, et al. A nutraceutical approach to menopausal complaints. Medicina. 2019;55(9):544. doi:10.3390/medicina55090544

8. Fisher C, Hickman L, Adams J, Sibbritt D. Cyclic perimenstrual pain and discomfort and Australian women's associated use of complementary and alternative medicine: a longitudinal study. $J$ Womens Health. 2018;27(1):40-50. doi:10.1089/jwh.2016.6253

9. Dias SFL, Pereira LCA. Scientific and technological prospection on transdermal formulations and complementary therapies for the treatment of primary dysmenorrhea. Expert Opin Ther Pat. 2019;29 (2):115-126. doi:10.1080/13543776.2019.1562547
10. Doğan H, Eroğlu S, Akbayrak T. The effect of kinesio taping and lifestyle changes on pain, body awareness and quality of life in primary dysmenorrhea. Complement Ther Clin Pract. 2020;39:101120. doi:10.1016/j.ctcp.2020.101120

11. Chen L, Tang L, Guo S, Kaminga AC, Xu H. Primary dysmenorrhea and self-care strategies among Chinese college girls: a cross-sectional study. BMJ Open. 2019;9(9):e026813. doi:10.1136/bmjopen-2018026813

12. Pattanittum P, Kunyanone N, Brown J, et al. Dietary supplements for dysmenorrhoea. Cochrane Database Syst Rev. 2016;2016(3). doi:10.1002/14651858.CD002124.pub2

13. Kim SD. Yoga for menstrual pain in primary dysmenorrhea: a meta-analysis of randomized controlled trials. Complement Ther Clin Pract. 2019;36(February):94-99. doi:10.1016/j.ctcp.2019.0 6.006

14. Rakhshaee Z. Effect of three yoga poses (cobra, cat and fish poses) in women with primary dysmenorrhea: a randomized clinical trial. J Pediatr Adolesc Gynecol. 2011;24(4):192-196. doi:10.1016/j. jpag.2011.01.059

15. McGovern CE, Cheung C. Yoga and quality of life in women with primary dysmenorrhea: a systematic review. J Midwifery Womens Health. 2018;63(4):470-482. doi:10.1111/jmwh. 12729

16. Fernández-Martínez E, Onieva-Zafra MD, Parra-Fernández ML. The impact of dysmenorrhea on quality of life among Spanish female university students. Int J Environ Res Public Health. 2019;16(5):713. doi:10.3390/ijerph16050713

17. Azima S, Bakhshayesh HR, Kaviani M, Abbasnia K, Sayadi M. Comparison of the effect of massage therapy and isometric exercises on primary dysmenorrhea: a randomized controlled clinical trial. J Pediatr Adolesc Gynecol. 2015;28(6):486-491. doi:10.1016/j. jpag.2015.02.003

18. Ortiz MI, Cortés-Márquez SK, Romero-Quezada LC, Murguía-Cánovas G, Jaramillo-Díaz AP. Effect of a physiotherapy program in women with primary dysmenorrhea. Eur $J$ Obstet Gynecol Reprod Biol. 2015;194:24-29. doi:10.1016/j.ejogrb.20 15.08.008

19. Armour M, Sinclair J, Chalmers KJ, Smith CA. Self-management strategies amongst Australian women with endometriosis: a national online survey. BMC Complement Altern Med. 2019;19(1). doi:10.1186/s12906-019-2431-x

20. Sultana A, Lamatunoor S, Begum M, Qhuddsia QN. Management of Usr-i-Tamth (menstrual pain) in unani (greco-islamic) medicine. J Evid Based Complement Alternat Med. 2017;22(2):284-293. doi:10.1177/2156587215623637

21. Pellow J, Nienhuis C. Medicinal plants for primary dysmenorrhoea: a systematic review. Complement Ther Med. 2018;37 (November2017):13-26. doi:10.1016/j.ctim.2018.01.001

22. Abdollahi NG, Mirghafourvand M, Mollazadeh S. The effects of fennel on menstrual bleeding: a systematic review and meta-analysis. $J$ Complement Integr Med. 2018;15(3):1-13. doi:10.1515/jcim-2017-0154

23. Hashemi SA, Azadeh S, Nouri BM, Navai RA. Review of pharmacological effects of Zataria multiflora Boiss. (thyme of Shiraz). Int J Med Sci Public Health. 2017;6(8):78-84.

24. Iravani M. Clinical effects of Zataria multiflora essential oil on primary dysmenorrhea. J Med Plants. 2009;8(30). doi:10.1016/ s1550-8579(06)80120-8

25. Shirvani MA, Motahari-Tabari N, Alipour A. The effect of mefenamic acid and ginger on pain relief in primary dysmenorrhea: a randomized clinical trial. Arch Gynecol Obstet. 2015;291 (6):1277-1281. doi:10.1007/s00404-014-3548-2

26. Jahangirifar M, Taebi M, Dolatian M. The effect of Cinnamon on primary dysmenorrhea: a randomized, double-blind clinical trial. Complement Ther Clin Pract. 2018;33(June):56-60. doi:10.1016/j. ctcp.2018.08.001 
27. Raisi Dehkordi Z, Hosseini Baharanchi FS, Bekhradi R. Effect of lavender inhalation on the symptoms of primary dysmenorrhea and the amount of menstrual bleeding: a randomized clinical trial. Complement Ther Med. 2014;22(2):212-219. doi:10.1016/j. ctim.2013.12.011

28. Pio IDSL, Lavor AL, Damasceno CMD, Menezes PMN, Silva FS, Maia GLA. Traditional knowledge and uses of medicinal plants by the inhabitants of the islands of the são francisco river, Brazil and preliminary analysis of rhaphiodon echinus (Lamiaceae). Braz J Biol. 2019;79(1):87-99. doi:10.1590/1519-6984.177447

29. Ghasemzadeh Rahbardar M, Amin B, Mehri S, Mirnajafi-Zadeh SJ, Hosseinzadeh $\mathrm{H}$. Anti-inflammatory effects of ethanolic extract of rosmarinus officinalis $\mathrm{L}$. and rosmarinic acid in a rat model of neuropathic pain. Biomed Pharmacother. 2017;86:441-449. doi:10.1016/j.biopha.2016.12.049

30. He X, Wang X, Fang J, et al. The genus achyranthes: a review on traditional uses, phytochemistry, and pharmacological activities J Ethnopharmacol. 2017;203:260-278. doi:10.1016/j.jep.2017.03.035

31. Jan S, Khan MR. Antipyretic, analgesic and anti-inflammatory effects of kickxia ramosissima. J Ethnopharmacol. 2016;182:90-100. doi:10.1016/j.jep.2016.02.020

32. Lee H, Choi TY, Myung CS, Lee JA, Lee MS. Herbal medicine (Shaofu Zhuyu decoction) for treating primary dysmenorrhea: a systematic review of randomized clinical trials. Maturitas. 2016;86:64-73. doi:10.1016/j.maturitas.2016.01.012

33. Moteetee A, Seleteng Kose L. Medicinal plants used in lesotho for treatment of reproductive and post reproductive problems. J Ethnopharmacol. 2016;194:827-849. doi:10.1016/j.jep.2016.10.062

34. Gao L, Jia C, Zhang H, Ma C. Wenjing decoction (herbal medicine) for the treatment of primary dysmenorrhea: a systematic review and meta-analysis. Arch Gynecol Obstet. 2017;296(4):679-689. doi:10.1007/s00404-017-4485-7

35. Jesuíno FW, Reis JP, Whitaker JCP. Effect of synadenium grantii and its isolated compound on dysmenorrhea behavior model in mice. Inflammopharmacology. 2019;27(3):613-620. doi:10.1007/s10787018-0501-1

36. Seo J, Lee H, Lee D, Jo H-G. Dangguijagyag-san for primary dysmenorrhea. Medicine (Baltimore). 2020;99(42):e22761. doi:10.1097/md.0000000000022761

37. Barassi G, Bellomo RG, Porreca A, Di Felice PA, Prosperi L, Saggini R. Somato-visceral effects in the treatment of dysmenorrhea: neuromuscular manual therapy and standard pharmacological treatment. $J$ Altern Complement Med. 2018;24(3):291-299. doi:10.1089/acm.2017.0182

38. Cha NH, Sok SR. Effects of auricular acupressure therapy on primary dysmenorrhea for female high school students in South Korea. J Nurs Scholarsh. 2016;48(5):508-516. doi:10.1111/jnu.12238

39. Abaraogu UO, Igwe SE, Tabansi-Ochiogu CS. Effectiveness of SP6 (Sanyinjiao) acupressure for relief of primary dysmenorrhea symptoms: a systematic review with meta- and sensitivity analyses. Complement Ther Clin Pract. 2016;25:92-105. doi:10.1016/j. ctcp.2016.09.003

40. Zhao MY, Zhang P, Li J, et al. Influence of de qi on the immediate analgesic effect of SP6 acupuncture in patients with primary dysmenorrhoea and cold and dampness stagnation: a multicentre randomised controlled trial. Acupunct Med. 2017;35(5):332-338. doi:10.1136/acupmed-2016-011228

41. Blödt S, Pach D, Eisenhart-Rothe SV. Effectiveness of app-based self-acupressure for women with menstrual pain compared to usual care: a randomized pragmatic trial. Am J Obstet Gynecol. 2018;218 (2):227.e1-227.e9. doi:10.1016/j.ajog.2017.11.570

42. Zhai SJ, Ruan Y, Liu Y, et al. Time-effective analgesic effect of acupressure ankle strip pressing wrist and ankle acupuncture point on primary dysmenorrhea: study protocol clinical trial (SPIRIT compliant). Med. 2020;99:12. doi:10.1097/MD.0000000000019496
43. Abaraogu UO, Igwe SE, Tabansi-Ochiogu CS, Duru DO. A systematic review and meta-analysis of the efficacy of manipulative therapy in women with primary dysmenorrhea. Explore. 2017;13 (6):386-392. doi:10.1016/j.explore.2017.08.001

44. Özgül S, Üzelpasaci E, Orhan C, Baran E, Beksaç MS, Akbayrak T. Short-term effects of connective tissue manipulation in women with primary dysmenorrhea: a randomized controlled trial. Complement Ther Clin Pract. 2018;33:1-6. doi:10.1016/j.ctcp.2018.07.007

45. Demirtürk F, Yilar Erkek Z, Alparslan Ö, Demirtürk F, Demir O, Inanir A. Comparison of reflexology and connective tissue manipulation in participants with primary dysmenorrhea. J Altern Complement Med. 2016;22(1):38-44. doi:10.1089/acm.2015.0050

46. Ruffini N, D’Alessandro G, Cardinali L, Frondaroli F, Cerritelli F. Osteopathic manipulative treatment in gynecology and obstetrics: a systematic review. Complement Ther Med. 2016;26:72-78. doi:10.1016/j.ctim.2016.03.005

47. Smith CA, Armour M, Zhu X, Li X, Lu ZY, Song J. Acupuncture for dysmenorrhoea. Cochrane Database Syst Rev. 2016;2016(4). doi:10.1002/14651858.CD007854.pub3

48. Shetty GB, Shetty B, Mooventhan A. Efficacy of acupuncture in the management of primary dysmenorrhea: a randomized controlled trial. $J$ Acupunct Meridian Stud. 2018;11(4):153-158. doi:10.1016/j. jams.2018.04.001

49. French L. Dysmenorrhea. Am Fam Physician. 2005;71(2):285-292.

50. Gaubeca-Gilarranz A, Fernández-De-Las-Peñas C, Medina-Torres JR, et al. Effectiveness of dry needling of rectus abdominis trigger points for the treatment of primary dysmenorrhoea: a randomised parallel-group trial. Acupunct Med. 2018;36(5):302-310. doi:10.1136/acupmed-2017-011566

51. Armour M, Dahlen HG, Zhu X, Farquhar C, Smith CA. The role of treatment timing and mode of stimulation in the treatment of primary dysmenorrhea with acupuncture: an exploratory randomised controlled trial. PLoS One. 2017;12(7):1-20. doi:10.1371/journal. pone. 0180177

52. Armour M, Smith CA. Treating primary dysmenorrhoea with acupuncture: a narrative review of the relationship between acupuncture "dose" and menstrual pain outcomes. Acupunct Med. 2016;34 (6):416-424. doi:10.1136/acupmed-2016-011110

53. Wang H, Cao Y, Jin X, et al. Effect of an acupuncture technique of penetrating through zhibian (BL54) to shuidao (ST28) with long needle for pain relief in patients with primary dysmenorrhea: a randomized controlled trial. Pain Res Manag. 2019;2019:1-6. doi: $10.1155 / 2019 / 7978180$

54. Sut N, Kahyaoglu-Sut H. Effect of aromatherapy massage on pain in primary dysmenorrhea: a meta-analysis. Complement Ther Clin Pract. 2017;27:5-10. doi:10.1016/j.ctcp.2017.01.001

55. Uysal M, Doğru HY, Sapmaz E, et al. Investigating the effect of rose essential oil in patients with primary dysmenorrhea. Complement Ther Clin Pract. 2016;24:45-49. doi:10.1016/j.ctcp.2016.05.002

56. Song J-A, Lee M-K, Min E, Kim M-E, Fike G, Hur M-H. Effects of aromatherapy on dysmenorrhea: a systematic review and meta-analysis. Int $J$ Nurs Stud. 2018;84:1-11. doi:10.1016/j. ijnurstu.2018.01.016

57. Charandabi SMA, Biglu MH, Rad KY. Effect of homeopathy on pain intensity and quality of life of students with primary dysmenorrhea: a randomized controlled trial. Iran Red Crescent Med J. 2016;18 (9):1-8. doi:10.5812/ircmj.30902

58. Reid R, Steel A, Wardle J, Adams J. Naturopathic medicine for the management of endometriosis, dysmenorrhea, and menorrhagia: a content analysis. J Altern Complement Med. 2019;25(2):202-226. doi:10.1089/acm.2018.0305

59. Navvabi Rigi S, Kermansaravi F, Navidian A, et al. Comparing the analgesic effect of heat patch containing iron chip and ibuprofen for primary dysmenorrhea: a randomized controlled trial. BMC Womens Health. 2012;12(1):25. doi:10.1186/1472-6874-12-25 
60. Shin Y Il, Kim NG, Park KJ, Kim DW, Hong GY, Shin BC. Skin adhesive low-level light therapy for dysmenorrhoea: a randomized, double-blind, placebo-controlled, pilot trial. Arch Gynecol Obstet. 2012;286(4):947-952. doi:10.1007/s00404-012-2380-9

61. Lee JM, Kim KH. Effect of near-infrared rays on female menstrual pain in Korea. Nurs Health Sci. 2017;19(3):366-372. doi:10.1111/ nhs. 12356

62. Abubakar U, Zulkarnain AI, Samri F, Hisham SR, Alias A, Ishak M. $\mathrm{CAM}$ and dysmenorrhoea in Malaysia undergraduates. $B M C$ Complement Altern Med. 2020;4:1-8.

63. Pakpour AH, Kazemi F, Alimoradi Z, Griffiths MD. Depression, anxiety, stress, and dysmenorrhea: a protocol for a systematic review. Syst Rev. 2020;9(1):65. doi:10.1186/s13643-020-01319-4

64. Bajalan Z, Moafi F, MoradiBaglooei M, Alimoradi Z. Mental health and primary dysmenorrhea: a systematic review. J Psychosom Obstet Gynaecol. 2018;1-10. doi:10.1080/0167482X.2018.1470619

65. Rodrigues AC, Gala S, Neves Â, et al. Dysmenorrhea in adolescents and young adults: prevalence, related factors and limitations in daily living. Acta Med Port. 2011;24(Suppl 2):383-392.

66. Payne LA, Rapkin AJ, Lung KC, Seidman LC, Zeltzer LK, Tsao JCI. Pain catastrophizing predicts menstrual pain ratings in adolescent girls with chronic pain. Pain Med. 2016;17(1):16-24. doi:10.1111/ pme.12869

67. Walsh TM, LeBlanc L, McGrath PJ. Menstrual pain intensity, coping, and disability: the role of pain catastrophizing. Pain Med. 2003;4 (4):352-361. doi:10.1111/j.1526-4637.2003.03039.x
68. Mellegård M, Grossi G, Soares JJF. A comparative study of coping among women with fibromyalgia, neck/shoulder and back pain. Int $J$ Behav Med. 2001;8(2):103-115. doi:10.1207/ S15327558IJBM0802_02

69. Keefe FJ, Lumley M, Anderson T, Lynch T, Carson KL. Pain and emotion: new research directions. J Clin Psychol. 2001;57 (4):587-607. doi:10.1002/jclp. 1030

70. Yilmaz B, Sahin N. The effects of a dysmenorrhea support program on university students who had primary dysmenorrhea: a randomized controlled study. J Pediatr Adolesc Gynecol. 2020;33(3):285-290. doi:10.1016/j.jpag.2019.12.008

71. Proctor M, Murphy PA, Pattison HM, Suckling J, Farquhar CM. Behavioural interventions for primary and secondary dysmenorrhoea. Cochrane Database Syst Rev. 2007;2007(3). doi:10.1002/14651858.CD002248.pub3

72. Stones RW, Mountfield J. Interventions for treating chronic pelvic pain in women. Cochrane Database Syst Rev. 2000;(4):CD000387. doi:10.1002/14651858.CD000387

73. De Sanctis V, Soliman A, Bernasconi S, et al. Primary dysmenorrhea in adolescents: prevalence, impact and recent knowledge. Pediatr Endocrinol Rev. 2015;13(2):512-520.

74. Dalpiaz O, Kerschbaumer A, Mitterberger M, Pinggera G, Bartsch G, Strasser H. Chronic pelvic pain in women: still a challenge. BJU Int. 2008;102(9):1061-1065 doi:10.1111/j.1464-410X.2008.07771. xsssssss

\section{Publish your work in this journal}

The International Journal of Women's Health is an international, peerreviewed open-access journal publishing original research, reports, editorials, reviews and commentaries on all aspects of women's healthcare including gynecology, obstetrics, and breast cancer. The manuscript management system is completely online and includes a very quick and fair peer-review system, which is all easy to use. Visit http://www.dovepress.com/testimonials.php to read real quotes from published authors. 